\title{
Real-world effectiveness and safety of omalizumab in patients with uncontrolled severe allergic asthma from the Czech Republic
}

\author{
Olga Růžičková Kirchnerová1, Tomáš Valena², Jakub Novosad³, Milan Teřrl', on behalf of Czech eXpeRience Study Group* \\ ${ }^{1}$ Department of Pneumology and Phthisiology, Charles University in Prague, Faculty of Medicine in Pilsen, Czech Republic \\ ${ }^{2}$ Novartis s.r.o., Prague, Czech Republic \\ ${ }^{3}$ Institute of Clinical Immunology and Allergy, Charles University in Prague, Faculty of Medicine in Hradec Kralove, University Hospital \\ in Hradec Kralove, Czech Republic
}

*Collaborators: J. Bystroň (University Hospital Olomouc), J. Chlumský (Thomayer Hospital Praha), D. Kindlová (University Hospital Brno), J. Klosová (University Hospital Ostrava), I. Krčmová (University Hospital Hradec Kralové), Z. Paráková (University Hospital Hradec Kralové), P. Pohunek (University Hospital in Motol), P. Popelková (University Hospital Ostrava), V. Sedlák (University Hospital Hradec Kralové), P. Vaník (Hospital České Budějovice), E. Vernerová (University Hospital in Motol), J. Zatloukal (University Hospital Olomouc)

Adv Dermatol Allergol 2019; XXXVI (1): 34-43 DOI: https://doi.org/10.5114/ada.2018.76606

\begin{abstract}
Introduction: This was a sub-group analysis of patients with uncontrolled persistent allergic asthma (AA) in the healthcare setting of the Czech Republic, from a global non-interventional, 2-year post-marketing, observational eXpeRience registry.

Aim: To evaluate the real-life effectiveness and safety of omalizumab.

Material and methods: Patients with uncontrolled persistent AA (currently defined by the Global Initiative for Asthma (GINA) as uncontrolled severe AA) who started omalizumab treatment 15 weeks before inclusion in the registry were analysed for physicians' global evaluation of treatment effectiveness (GETE), asthma symptoms, corticosteroid use, exacerbation rate, asthma control, quality of life, healthcare utilisation and safety during a 24-month observation period.

Results: One hundred and fourteen patients from the Czech Republic were enrolled in the eXpeRience registry. A total of $88.9 \%$ of the patients were evaluated as responders to omalizumab according to the GETE assessment at week 16. From baseline to month 24: mean change in forced expiratory volume in $1 \mathrm{~s}\left(\mathrm{FEV}_{1}\right)$ was $137 \mathrm{ml}$ and the daily dose of OCS decreased (11.6 mg to $6.4 \mathrm{mg}$ prednisolone equivalent); the percentage of patients with no severe clinically significant exacerbations increased (29.5\% to 95.1\%); Asthma Control Test scores improved (12.4 to 17.3) and mean total number of days of asthma-related medical healthcare use decreased ( 6.8 days to 0.4 days). Conclusions: The results of this subgroup analysis support the evidence that add-on omalizumab therapy is effective and well tolerated for management of patients with uncontrolled persistent AA in the Czech Republic. Global evaluation of treatment effectiveness assessment is a reliable predictor of long-term response to omalizumab treatment.
\end{abstract}

Key words: observational registry, omalizumab, real-world study, allergic asthma, asthma control, exacerbations.

\section{Introduction}

Worldwide prevalence of asthma varies between different regions and countries in a wide range from $1 \%$ to $16 \%$ [1]. The prevalence of asthma in the European Union (EU) was reported to be in the range 5-16\% [2]. Although exact data on the prevalence of asthma in the Czech Republic (population of about 10 million) are not known, it was estimated that $8 \%$ of the Czech population, i.e. around 800 thousand people, suffered from asthma [1]. Nearly half of the estimated patients were reported to be under the care of allergologists and/or pulmonologists [3].

Many patients with severe asthma remain uncontrolled despite receiving combinations of high-dose inhaled corticosteroids (ICS) and long-acting $\beta_{2}$-agonists (LABA) in addition to other medications (leukotriene receptor antagonists, theophylline) [4], and continue to ex-

Address for correspondence: Tomáš Valena MSc, Novartis s.r.o, Na Pankráci 1724/129, 14000 Praha 4, Czech Republic, phone: +420 225775 111, +420 734284 002, fax: +420 225775 222, e-mail: tomas.valena@novartis.com Received: 16.04.2018, accepted: 14.05.2018. 
perience asthma symptoms, lifestyle restrictions leading to poor quality of life and even frequent exacerbations needing emergency care [5].

Omalizumab is a humanized anti-immunoglobulin $\mathrm{E}$ (IgE) monoclonal antibody approved in the EU for patients with severe persistent allergic asthma aged $\geq 6$ years who are inadequately controlled with high-dose ICS and LABA. As an add-on treatment, omalizumab has been shown to improve long-term asthma control and lung function, and reduce the use of corticosteroids, rescue medication and frequency of exacerbations in patients with persistent allergic asthma [6-8]. Supporting the results from clinical studies, real-world studies conducted in various European countries also reported improvement in asthma control and quality of life of patients after treatment with omalizumab [9-12]. To strengthen the evidence for omalizumab, a 2-year, global, post-marketing observational registry (eXpeRience) was conducted $[13,14]$.

\section{Aim}

Here, we report the outcomes of add-on omalizumab treatment in patients from the Czech Republic enrolled in the expeRience registry.

\section{Material and methods}

\section{Design and patients}

expeRience was a 2-year, international, post-marketing, non-interventional, multicentre (14 countries in Europe, the Americas and Asia), open-label, single-arm, observational registry which collected safety and effectiveness data from patients treated with add-on omalizumab for uncontrolled persistent allergic (IgE-mediated) asthma $[13,14]$. The dose of omalizumab administered was determined following the European Medicines Agency Summary of Product Characteristics [15].

Patients were eligible for inclusion if they met the labelling and local re-imbursement requirements for omalizumab use and had received omalizumab between 15 weeks and 18 months prior to the start of the registry. All patients who provided informed consent entered the registry. Patients were excluded if they were participating in any other clinical study or had received omalizumab in the past 18 months. Detailed inclusion and exclusion criteria have been described previously [14].

The registry design was reviewed by independent ethics committees or institutional review boards at each centre, and the registry itself was conducted in accordance with the Declaration of Helsinki.

The current study was a post-hoc analysis of the global expeRience registry.

\section{Assessments}

After enrolment in the registry, patients' baseline data (medical history, asthma status and control and medical resource use in the last 12 months before the start of the therapy) were collected retrospectively from their medical records. Patients were followed up for 2 years after initiation of omalizumab treatment and data were collected for analysis at week $16 \pm 1$ week, and months $8,12,18$, and 24 . Data reported in this analysis include patient response to therapy (as determined by physician's global evaluation of treatment effectiveness (GETE)), lung function ( $\mathrm{FEV}_{1} \%$ predicted and peak expiratory flow (PEF)), clinical symptoms (day- and night-time symptoms), use of rescue medication, use of oral and inhaled corticosteroids, number of clinically significant and severe asthma exacerbations, Asthma Control Test $(A C T)$, patients' health-related quality of life (mini-Asthma Quality of Life Questionnaire (mini-AQLQ)), medical healthcare resource use (hospitalisations, emergency room (ER) visits or unscheduled doctor visits or interventions), number of missed days of work/school, serious adverse events (SAEs) and deaths [13, 16, 17].

A clinically significant exacerbation was defined as any worsening of asthma considered by the treating physician to require systemic corticosteroids, and were recognised as severe if there was a reduction in PEF to $<60 \%$ of the patient's predicted or personal best. Data on exacerbations were annualised; that is, for month 12 , rates were derived from week 16 , month 8 and month 12 data; for month 24 , rates were derived from data collected at months 18 and 24 [14].

Global evaluation of treatment effectiveness analysis was performed at week $16 \pm 1$ week by physicians. Patients were rated on a 5-point scale: 1 - excellent (complete control of asthma), 2 - good (marked improvement), 3 - moderate (discernible, but limited improvement), 4 - poor (no appreciable change), and 5 - worsening (overall deterioration of asthma control). Patients with an "excellent" or "good" response were considered responders, those with "moderate", "poor" or "worsening" as non-responders [18].

The ACT is a patient-reported 5-item questionnaire that assesses shortness of breath, asthma symptoms, rescue medication use, the effect of asthma on daily functioning, and self-rating of asthma control. Each item includes responses with values from 1 to 5 to give a final ACT score of 5 (poorly controlled asthma) to 25 (wellcontrolled asthma). An improvement of $\geq 3$ units in the ACT score is considered to be the minimal important difference $[17,19]$.

The mini-AQLQ is a 15-item questionnaire (a shorter version of the 32-item AQLQ) covering four domains: symptoms (5 items), activity limitation (4 items which are patient specific), emotional function (3 items) and environmental stimuli (3 items). Patients were asked to respond to each question based on their experience during the previous two weeks, on a 7-point scale (0 - no impairment to 6 - maximum impairment). The overall miniAQLQ score is the mean of all 15 responses. A change in 
mini-AQLQ score of $\geq 0.5$ is considered to be the minimal clinically important difference [20, 21].

\section{Statistical analysis}

All effectiveness variables were analysed using the intent-to-treat (ITT) population, which consisted of all pa-

Table 1. Baseline demographics and clinical characteristics (safety population) $(N=112)$

\begin{tabular}{|c|c|}
\hline Variable & Results \\
\hline \multicolumn{2}{|l|}{ Age group, $n(\%)$ [years]: } \\
\hline$<12$ & $1(0.9)$ \\
\hline $12-17$ & $3(2.7)$ \\
\hline $18-64$ & $102(91.1)$ \\
\hline$\geq 65$ & $6(5.4)$ \\
\hline Age, mean \pm SD [years] & $44.0 \pm 13.0$ \\
\hline Female, $n(\%)$ & $68(60.7)$ \\
\hline \multicolumn{2}{|l|}{ Race, $n(\%)$ : } \\
\hline Caucasian & $112(100.0)$ \\
\hline Other & $0(0)$ \\
\hline Body weight, mean \pm SD [kg] & $77.3 \pm 15.9$ \\
\hline Baseline IgE level, mean \pm SD [IU/ml] & $290.3 \pm 262.5$ \\
\hline $\begin{array}{l}\text { Duration of allergic asthma, } \\
\text { mean } \pm \text { SD [years] }\end{array}$ & $18.5 \pm 12.4$ \\
\hline $\mathrm{FEV}_{1} \%$ predicted, mean $\pm \mathrm{SD}$ & $61.0 \pm 16.3$ \\
\hline $\begin{array}{l}\text { Positive skin-prick test/RAST for } \\
\text { perennial aeroallergens, } n(\%)\end{array}$ & $105(93.8)$ \\
\hline \multicolumn{2}{|l|}{ Specification, $n(\%)^{\mathrm{a}}$ : } \\
\hline Dust mites & $81(72.3)$ \\
\hline Cat dander & $47(42.0)$ \\
\hline Dog dander & $50(44.6)$ \\
\hline Cockroaches & $3(2.7)$ \\
\hline Others & $19(17.0)$ \\
\hline $\begin{array}{l}\text { History of allergy to seasonal } \\
\text { aeroallergens, } n(\%)\end{array}$ & $93(83.0)$ \\
\hline \multicolumn{2}{|l|}{ Smoking history, $n(\%)$ : } \\
\hline Never smoked & $85(75.9)$ \\
\hline Ex-smoker & $26(23.2)$ \\
\hline Current smoker & $1(0.9)$ \\
\hline \multicolumn{2}{|l|}{ Asthma clinical symptoms, $n$ (\%): } \\
\hline Daytime asthma symptoms & $109(97.3)$ \\
\hline Limitations of activities & $110(98.2)$ \\
\hline Nocturnal symptoms/awakenings & $96(85.7)$ \\
\hline \multicolumn{2}{|l|}{ Asthma control, $n(\%)^{\mathrm{b}}$ : } \\
\hline Partly controlled & $27(24.1)$ \\
\hline Uncontrolled & $85(75.9)$ \\
\hline
\end{tabular}

aMultiple entries possible, b/nvestigator's assessment, IgE-immunoglobulin $E$ $R A S T$ - radio-allergo-sorbent test, $S D$ - standard deviation. tients from the Czech Republic who enrolled in the whole registry and received at least one dose of omalizumab and who had at least one post-baseline efficacy assessment. For analyses at specific time-points, all patients with available data at that time-point were considered for the analysis. The safety population consisted of all patients from the Czech Republic who enrolled in the registry and who received at least one dose of omalizumab and had at least one post-baseline safety assessment. The safety population was used for collection of data on demographics, baseline characteristics and safety evaluation. Descriptive statistics are expressed as means with standard deviations (SD) or as frequencies. Repeated measures were treated using a general linear model for repeated measures (GLM-RM) or the Friedman ANOVA in cases of normality assumption violation. Frequencies were analysed using the $\chi^{2}$ test. Survival analysis was performed using the Kaplan-Meier algorithm (with mean survival time calculation) or Cox regression (with hazard ratio (HR) with 95\% confidence interval calculation). The $p$-value $<0.05$ was considered as statistically significant. Data were analysed by IBM SPSS Statistics for Windows, Version 22.0. Armonk, NY: IBM Corp.

\section{Results}

From a total of 943 patients enrolled in the eXpeRience registry, 114 patients originated from the Czech Republic. The ITT and safety population included 112 patients each. Patients' demographics and baseline clinical characteristics are presented in Table 1. Overall, 17 (14.9\%) patients discontinued the registry; reasons for discontinuation included lost to follow-up $(n=5)$, patient withdrew consent $(n=3)$, death $(n=1)$, and other reasons $(n=8) ; 7$ patients from the Czech subgroup had missing data or unresolved data queries. At the end of the registry (month 24), data related to treatment were available for 81 patients from the Czech Republic.

\section{Physician's global evaluation of treatment effectiveness}

Ninety patients had a GETE assessment at week $16 \pm 1$ week, of whom $88.9 \%(n=80)$ were responders (excellent and good response to omalizumab therapy in $7.8 \%$ $(n=7)$ and $81.1 \%(n=73)$ of patients, respectively), and $11.1 \%(n=10)$ were non-responders (Figure 1$)$. For GETE assessed at any time-point, $85.7 \%(n=96)$ of patients were responders whereas $14.3 \%(n=16)$ were non-responders.

The effect of GETE on health care use risk is described in the section Healthcare utilisation and missed work days.

\section{Lung function}

Treatment with omalizumab resulted in improvement in $\mathrm{FEV}_{1}$ with a mean change from baseline $(\mathrm{ml})$ of 205 
( $n=105), 215(n=88), 273(n=87), 200(n=85)$ and 137 $(n=80)$ at week 16 and months $8,12,18$ and 24 , respectively. The PEF was also improved with a mean change from baseline $(\mathrm{l} / \mathrm{min})$ of $11.01(n=82), 18.38(n=66)$, $32.82(n=65), 25.18(n=63)$ and $21.85(n=60)$ at week 16 and months $8,12,18$ and 24 , respectively.

\section{Asthma symptoms and rescue medication use}

Omalizumab reduced the number of patients experiencing daytime symptoms, nocturnal symptoms/awakenings, activity limitations and rescue medication use in the week before months 12 and 24 versus baseline. During the week prior to the baseline visit, 108 (96.4\%) patients had been using rescue medication; this was reduced to $61(75.3 \%)$ patients at month 24. Mean use of rescue medication (short-acting $\beta_{2}$-agonists) decreased from 5.2 days/week at baseline to 2.9 days/week at month 24 of omalizumab treatment (Table 2).

\section{Oral corticosteroid use}

Oral corticosteroids (OCS) were used as maintenance therapy in 38 patients (33.9\%) with a mean total daily dose of $11.6 \mathrm{mg}$ (prednisolone equivalent). The proportion of patients on OCS maintenance therapy compared to baseline decreased by $50 \%(n=19)$ and $52.6 \%(n=18)$ patients at months 12 and 24, respectively. The mean total daily dose of OCS (in prednisolone equivalent, $\mathrm{mg}$ ) in the population using OCS maintenance therapy at baseline decreased to $6.4 \mathrm{mg}$ at months 12 and 24 (Figure 2).

\section{Exacerbations}

The mean number of clinically significant exacerbations decreased with omalizumab from $5.7(n=104)$ at baseline to $1.1(n=89)$ and $0.7(n=80)$ and severe exacerbations decreased from $2.2(n=105)$ at baseline to $0.2(n=89)$ and $0.1(n=81)$ at months 12 and 24, respectively (Table 3). Furthermore, the proportion of patients with no clinically significant/severe exacerbations was markedly increased with omalizumab therapy to $56.2 \%$ $(n=50) / 89.9 \%(n=80)$ and $63.0 \%(n=51) / 95.1 \%$ $(n=77)$ at months 12 and 24 , respectively. The mean numbers of clinically significant exacerbations and severe exacerbations in the population using OCS maintenance therapy at baseline are presented in Figure 2.

\section{Asthma control and health-related quality of life}

After 12 and 24 months of omalizumab treatment, 92.1\% $(n=82)$ and $87.7 \%(n=71)$ of patients reported controlled or partly controlled asthma, which is substantially higher than that at baseline $(24.1 \%$; $n=27)$, as per physicians' assessment (Table 3 ).

The ACT scores increased from $12.4(n=103)$ at baseline to $17.3(n=84)$ and $17.3(n=75)$ at months 12 and 24 , respectively. Clinically meaningful improvements from baseline in ACT scores were observed with omalizumab

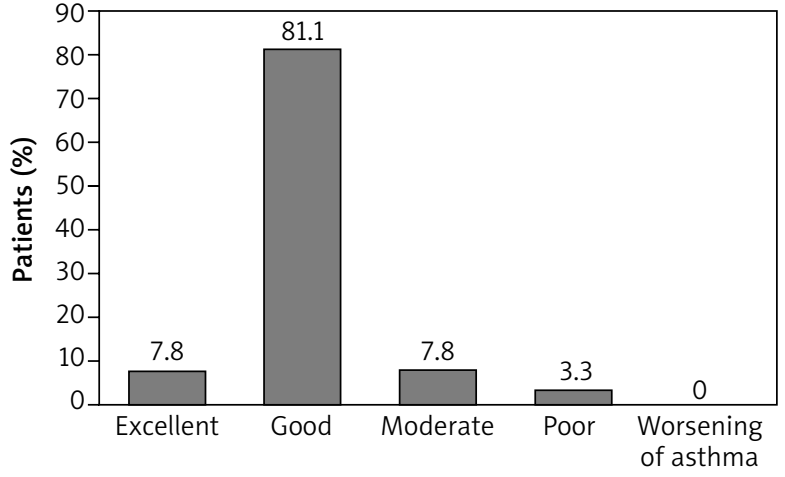

Figure 1. Physician's global evaluation of treatment effectiveness (GETE) at week $16 \pm 1$ weeks $(n=90)$

treatment at months 12 (mean change: 4.3 points, $n=81$ ) and 24 (mean change: 4.3 points, $n=73$ ) (Table 3).

In OCS maintenance therapy at baseline, mean ACT score increased from baseline by $27.7 \%$ and $29.9 \%$ at months 12 and 24, respectively (Figure 2).

Similar improvements were observed in patient health-related quality of life assessed using the miniAQLQ. Mean changes of 0.8 and 0.94 points in the mini-AQLQ from baseline werer observed at months 12 $(n=38)$ and $24(n=32)$, respectively. Overall, $60.5 \%$ $(n=23)$ and $65.6 \%(n=21)$ of patients achieved clinically meaningful improvements in the mini-AQLQ score versus baseline (Table 3).

\section{Healthcare utilisation and missed work days}

Asthma-related total medical healthcare use (hospitalisations and ER/unscheduled doctor visits) per patient and missed days of work due to asthma were decreased with add-on treatment with omalizumab at months 12 and 24 compared with baseline (Table 3). The mean \pm SD total number of days of asthma-related medical healthcare use per patient decreased from $6.8 \pm 10.0$ during the 12-month pre-treatment period $(n=99)$ to $0.8 \pm 2.1$ and $0.4 \pm 1.2$ at months $12(n=88)$ and 24 $(n=80)$, respectively with omalizumab. Thus, healthcare resource utilisation was reduced compared with baseline by $88.2 \%$ and $94.1 \%$ at months 12 and 24 , respectively.

The effect of GETE on medical healthcare use risk was calculated using Cox regression (overall model evaluation $p<0.001)$. The reference value was defined as poor responders. The calculated hazard ratio (HR) for moderate responders was 0.26 ( $p=0.04,95 \% \mathrm{Cl}: 0.072-0.937)$, HR (good responder) $=0.168(p<0.001,95 \% \mathrm{Cl}: 0.067-$ 0.462 ) and HR (excellent responder) $=0.117$ ( $p=0.01$, $95 \% \mathrm{Cl}: 0.023-0.599)$. We did not register any case of worsening asthma during the study period (Figure 3). Thus in contrast to poor responders, excellent, good and moderate responders had a reduced risk of healthcare use by $88.3 \%, 83.2 \%$ and $74 \%$, respectively. 
Table 2. Effect of omalizumab on asthma symptoms, rescue medication and corticosteroid use

\begin{tabular}{|c|c|c|c|}
\hline \multirow[t]{2}{*}{ Parameter } & \multicolumn{3}{|c|}{ Czech Republic } \\
\hline & $\begin{array}{l}\text { Baseline } \\
(n=112)\end{array}$ & $\begin{array}{c}\text { Month } 12 \\
(n=89)\end{array}$ & $\begin{array}{c}\text { Month } 24 \\
(n=81)\end{array}$ \\
\hline \multicolumn{4}{|l|}{ Daytime symptoms*: } \\
\hline \multicolumn{4}{|l|}{ Number of days: } \\
\hline Mean (SD) & $5.5(2.2)$ & $2.6(2.4)$ & $2.6(2.5)$ \\
\hline$N(\%)$ & $109(97.3)$ & $72(80.9)$ & $63(77.8)$ \\
\hline \multicolumn{4}{|l|}{ Change in number of days: } \\
\hline Mean (SD) & - & $-2.7(2.5)$ & $-2.7(2.6)$ \\
\hline$N$ & & 88 & 81 \\
\hline \multicolumn{4}{|l|}{ Nocturnal symptoms/awakening*: } \\
\hline \multicolumn{4}{|l|}{ Number of days: } \\
\hline Mean (SD) & $3.2(2.4)$ & $1.1(1.5)$ & $0.9(1.5)$ \\
\hline$N(\%)$ & $96(85.7)$ & $44(49.4)$ & $34(42.0)$ \\
\hline \multicolumn{4}{|l|}{ Change in number of days: } \\
\hline Mean (SD) & - & $-2.0(2.4)$ & $-2.3(2.1)$ \\
\hline$N$ & & 89 & 81 \\
\hline \multicolumn{4}{|l|}{ Limitations of activities*: } \\
\hline \multicolumn{4}{|l|}{ Number of days: } \\
\hline Mean (SD) & $5.0(2.1)$ & $2.2(2.0)$ & $2.2(2.2)$ \\
\hline$N(\%)$ & $110(98.2)$ & $72(80.9)$ & $57(70.4)$ \\
\hline \multicolumn{4}{|l|}{ Change in number of days: } \\
\hline Mean (SD) & - & $-2.8(2.3)$ & $-2.9(2.4)$ \\
\hline$N$ & & 87 & 80 \\
\hline \multicolumn{4}{|l|}{ Rescue medication use*: } \\
\hline \multicolumn{4}{|l|}{ Number of days: } \\
\hline Mean (SD) & $5.2(2.4)$ & $3.0(2.8)$ & $2.9(2.8)$ \\
\hline$N(\%)$ & $108(96.4)$ & $70(78.7)$ & $61(75.3)$ \\
\hline \multicolumn{4}{|l|}{ Change in number of days: } \\
\hline Mean (SD) & - & $-2.1(2.6)$ & $-2.1(2.7)$ \\
\hline$N$ & & 89 & 80 \\
\hline \multicolumn{4}{|l|}{ ICS usage: } \\
\hline Patients on ICS, $n$ (\%) & $111(99.1)$ & $87(97.8)$ & $78(96.3)$ \\
\hline Total ICS daily dose (beclomethasone dipropionate equivalent $\mu \mathrm{g}$ ), mean (SD) & $2196.4(1162.9)$ & $1947.2(876.1)$ & $1886.4(945.4)$ \\
\hline Number of patients with reduced ICS dose, $n(\%)$ & - & $21(23.6)$ & $21(25.9)$ \\
\hline
\end{tabular}

*Data are presented for the presence of these outcomes in the week before baseline, month 12, and month 24. ICS - inhaled corticosteroids, SD - standard deviation.

The mean \pm SD number of days of missed work due to asthma decreased from $24.3 \pm 27.4$ at baseline $(n=43)$ to $2.4 \pm 6.4$ and $1.7 \pm 5.1$ at months $12(n=43)$ and $24(n=38)$, respectively. Mean number of days of missed work due to asthma decreased by $90.1 \%$ and $93.0 \%$ after months 12 and 24, respectively.

The mean \pm SD number of asthma-related hospitalisations decreased from $0.5 \pm 1.2$ in the 12 -month pre-treatment period $(n=105)$ to $0.0 \pm 0.0$ at month 12 $(n=89)$ and $0.0 \pm 0.2$ at month $24(n=81)$. The mean \pm SD number of days stayed in hospital due to asthma decreased from $3.3 \pm 9.8$ in the 12 -month pre-treatment period $(n=100)$ to $0.3 \pm 2.0$ at month $12(n=89)$ and to $0.2 \pm 2.2$ at month $24(n=81)$. Overall, $100 \%$ and $98.8 \%$ of patients were free of asthma-related hospitalizations at months $12(n=89)$ and $24(n=80)$, respectively. 
Table 3. Asthma control, quality of life, exacerbations and asthma related medical healthcare use after omalizumab treatment

\begin{tabular}{|c|c|c|c|}
\hline \multirow[t]{2}{*}{ Parameter } & \multicolumn{3}{|c|}{ Czech Republic } \\
\hline & Baseline & Month 12 & Month 24 \\
\hline \multicolumn{4}{|l|}{ Total number of clinically significant asthma exacerbations: } \\
\hline$N$ & 104 & 89 & 80 \\
\hline Mean $(\mathrm{SD})^{*}$ & $5.7(7.4)$ & $1.1(1.8)$ & $0.7(1.2)$ \\
\hline \multicolumn{4}{|l|}{ Number of severe clinically significant asthma exacerbations: } \\
\hline$N$ & 105 & 89 & 81 \\
\hline Mean $(\mathrm{SD})^{*}$ & $2.2(3.3)$ & $0.2(0.7)$ & $0.1(0.3)$ \\
\hline Patient's level of asthma control': & $n=112$ & $n=89$ & $n=81$ \\
\hline Controlled, $n(\%)$ & $0(0.0)$ & $22(24.7)$ & $20(24.7)$ \\
\hline Partly controlled, $n(\%)$ & $27(24.1)$ & $60(67.4)$ & $51(63.0)$ \\
\hline Uncontrolled, $n$ (\%) & $85(75.9)$ & $7(7.9)$ & $10(12.3)$ \\
\hline \multicolumn{4}{|l|}{ ACT overall score: } \\
\hline$N$ & 103 & 84 & 75 \\
\hline Mean (SD) & $12.4(4.1)$ & $17.3(5.0)$ & $17.3(5.4)$ \\
\hline \multicolumn{4}{|l|}{ Change from baseline in ACT score: } \\
\hline$N$ & - & 81 & 73 \\
\hline Mean (SD) & & $4.3(4.0)$ & $4.3(3.8)$ \\
\hline \multicolumn{4}{|l|}{ Mini-AQLQ overall score: } \\
\hline$N$ & 46 & 45 & 41 \\
\hline Mean (SD) & $3.62(1.0)$ & $4.56(1.4)$ & $4.67(1.4)$ \\
\hline \multicolumn{4}{|l|}{ Change from baseline in mini-AQLQ overall score: } \\
\hline$N$ & - & 38 & 32 \\
\hline Mean (SD) & & $0.80(1.0)$ & $0.94(0.8)$ \\
\hline$\geq 0.5$ improvement, $n(\%)$ & & $23(60.5)$ & $21(65.6)$ \\
\hline \multicolumn{4}{|c|}{ Total number of days of asthma related medical healthcare use per patient $t^{\ddagger}$ : } \\
\hline N & 99 & 88 & 80 \\
\hline Mean (SD) & $6.8(10.0)$ & $0.8(2.1)$ & $0.4(1.2)$ \\
\hline \multicolumn{4}{|l|}{ Number of days missed work due to asthma: } \\
\hline N & 43 & 43 & 38 \\
\hline Mean (SD) & $24.3(27.4)$ & $2.4(6.4)$ & $1.7(5.1)$ \\
\hline \multicolumn{4}{|l|}{ Number of asthma related hospitalisations: } \\
\hline$N$ & 105 & 89 & 81 \\
\hline Mean (SD) & $0.5(1.2)$ & $0.0(0.0)$ & $0.0(0.2)$ \\
\hline Patients without asthma related hospitalizations, $n(\%)$ & $77(68.8)$ & $89(100.0)$ & $80(98.8)$ \\
\hline \multicolumn{4}{|l|}{ Total number of days stayed in hospital due to asthma: } \\
\hline$N$ & 100 & 89 & 81 \\
\hline Mean (SD) & $3.3(9.8)$ & $0.0(0.0)$ & $0.2(2.2)$ \\
\hline
\end{tabular}

${ }^{*}$ Baseline data includes the data during last 1 year before the start of the therapy, 'as per investigators' assessment, "includes hospitalizations, and emergency room/unscheduled doctor visits. ACT - Asthma Control Test, AQLQ - Asthma Quality of Life Questionnaire, SD - standard deviation. 


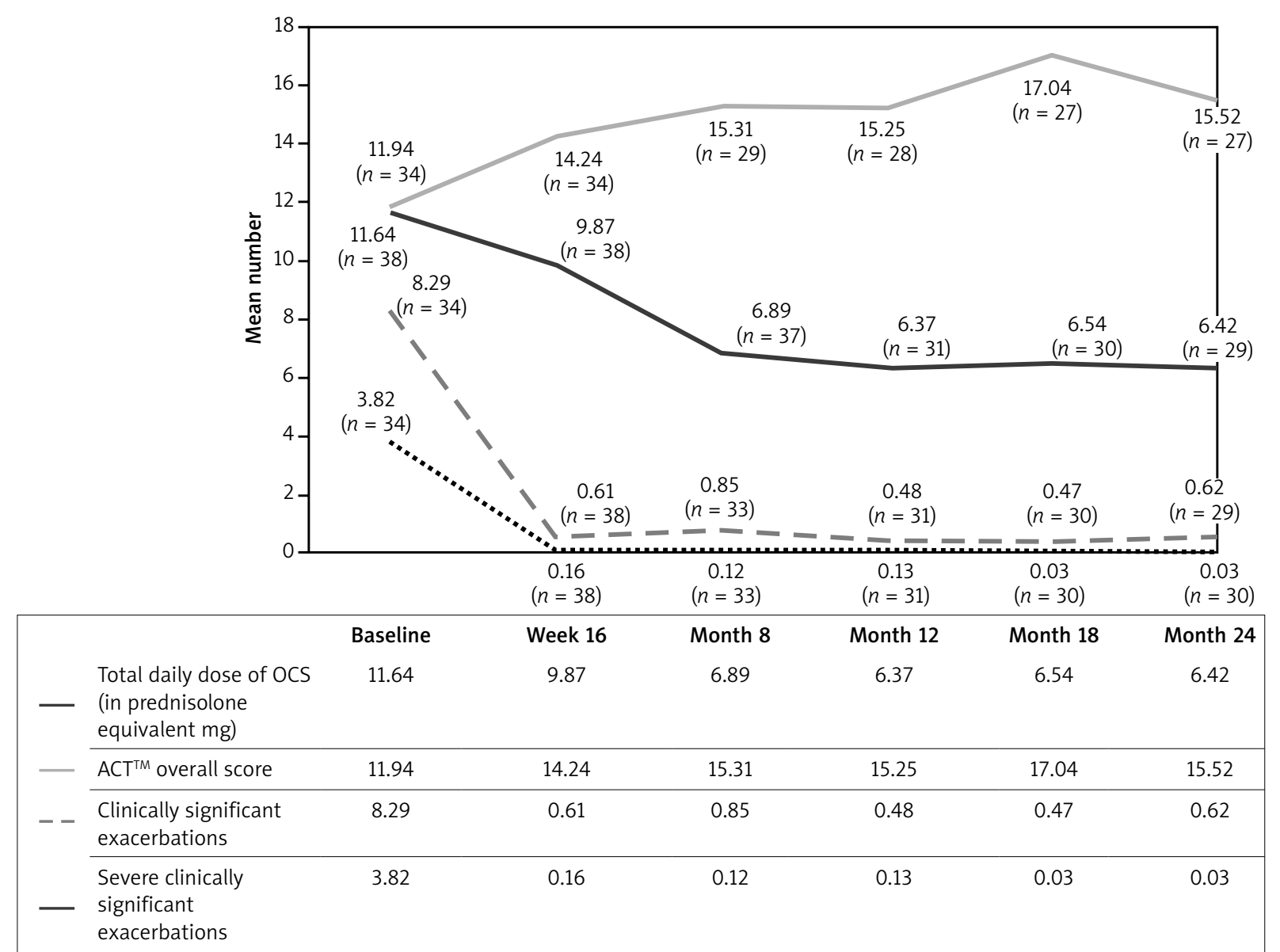

Values for OCS and ACT are for the week before the specified time points. Values for exacerbations at baseline are for 12 months prior to the start of omalizumab treatment. OCS - oral corticosteroids, ACT - Asthma Control Test, $N$-number of evaluable patients.

Figure 2. Effect of omalizumab on corticosteroid therapy, asthma control and exacerbations in OCS maintenance therapy population $(n=38)$

\section{Serious adverse events}

A total of 11 serious adverse events (SAEs) were reported in the 112 patients from the safety population. Four patients experienced each SAE in the 2-year observational period in the Czech Republic. These SAEs (hepatic failure, pneumonia in right lung, pulmonary embolism, sigmoid diverticulitis) were not suspected to be study-related. One patient experienced cumulated AE (headache, dizziness, flushing, hyperhidrosis, tremor, and dry mouth), which was considered as an SAE, and this was suspected to be study-related, leading to treatment withdrawal. One death occurred in this study group during the 2-year observation period (sudden death, due to viral endocarditis), which was reported as not being related to the treatment.

\section{Discussion}

The eXpeRience registry was designed to collect data on the real-world effectiveness and safety of omalizumab in patients with uncontrolled persistent allergic asthma
[14]. Results from this international registry provide data on patient demographics and baseline characteristics, treatment effectiveness and safety after 24 months of add-on omalizumab therapy $[13,14]$. The baseline demographics and clinical characteristics of the Czech Republic sub-population were comparable with the overall eXpeRience registry patient population.

Treatment effectiveness in patients from the Czech Republic was demonstrated by using GETE at $16 \pm 1$ weeks. The investigators rated the majority of patients as responders to omalizumab therapy (excellent/good; $88.9 \%$ ), which was $27.2 \%$ higher than that in the overall population (69.9\%) [13].

In the Czech Republic, physicians refer patients with asthma who are not able to achieve complete control even with GINA step 4 treatment to one of the specialised sites of the National Centres for Severe Asthma (NCTA) [22]. Each individual patient is re-evaluated in these centres for asthma diagnosis, comorbidities and complicating factors. Based on the consensus of both 
an allergologist and a pulmonologist from each centre, biological therapy could be initiated. Only NCTA centres are eligible to indicate biological treatment with anti-lgE antibody (omalizumab) in the Czech Republic [22, 23].

After 16 weeks, and 8, 12, 18 and 24 months of treatment with omalizumab, lung function assessed as mean change from baseline in $\mathrm{FEV}_{1}$ improved by $205 \mathrm{ml}$, $215 \mathrm{ml}, 273 \mathrm{ml}, 200 \mathrm{ml}$ and $137 \mathrm{ml}$, respectively. The effect of add-on omalizumab to high-dose ICS/LABA on FEV $\mathrm{F}_{1}$ is comparable to what was reported for add-on tiotropium in patients with poorly controlled asthma [24].

Omalizumab add-on treatment in the Czech Republic sub-population resulted in considerable reductions in the daytime and nocturnal symptoms, limitations of activities, and rescue medication use, which was comparable with results seen in the overall population of the expeRience registry and other real-life studies [10, 11, 13, 25-29]. There was also a considerable reduction from baseline in the number of patients on OCS maintenance therapy $(n=38)$ to 24 months after the treatment $(n=18)$ along with a $44.8 \%$ reduction in the total daily dose from $11.6 \mathrm{mg}$ at baseline (prednisolone equivalent) to $6.4 \mathrm{mg}$ at month 24. The effect on OCS maintenance dose was associated with a substantial increase in ACT score. In this regard, it is worth noting that omalizumab add-on treatment is recommended in GINA as the preferred choice over OCS in step 5 [1]. Severe asthma patients require long-term treatment, and withdrawal from omalizumab may cause loss of asthma control and increase exacerbations and the OCS maintenance dose [29-31].

From the results presented in this report, it is evident that omalizumab add-on treatment reduced the number of clinically significant exacerbations (annualised) from 5.7 at pre-treatment to 0.7 after 24 months of treatment in the Czech Republic sub-population. These results were similar to those observed in the global registry (4.9 to 0.6), Czech Anti-IgE Registry (CAR) and other real-life data with up to 9 years of follow-up $[9,13,28,29,32$, 33]. Additionally, clinically meaningful improvements in asthma control (ACT; mean change from baseline of 4.3 units) and patient health-related quality of life (miniAQLQ; mean change from baseline of 0.94 units) were also observed. These results are supported by the results from various other studies (ACT improvement of 5.01 and AQLQ improvement of 0.91 from baseline) [7, 34]. Based on the investigator assessment in the Czech Republic, a higher percentage of patients had controlled or partly controlled asthma at month 12 versus baseline (92.1\% vs. $24.1 \%$ ) upon treatment with omalizumab. These results were higher than those seen in the overall population of the expeRience registry and symptoms remained controlled at month 24. Asthma-related healthcare resource utilisation and days of missed work due to asthma were also substantially reduced with omalizumab treatment in the Czech Republic sub-population. Reliability of GETE assessment at $16 \pm 1$ weeks as a predictor of healthcare

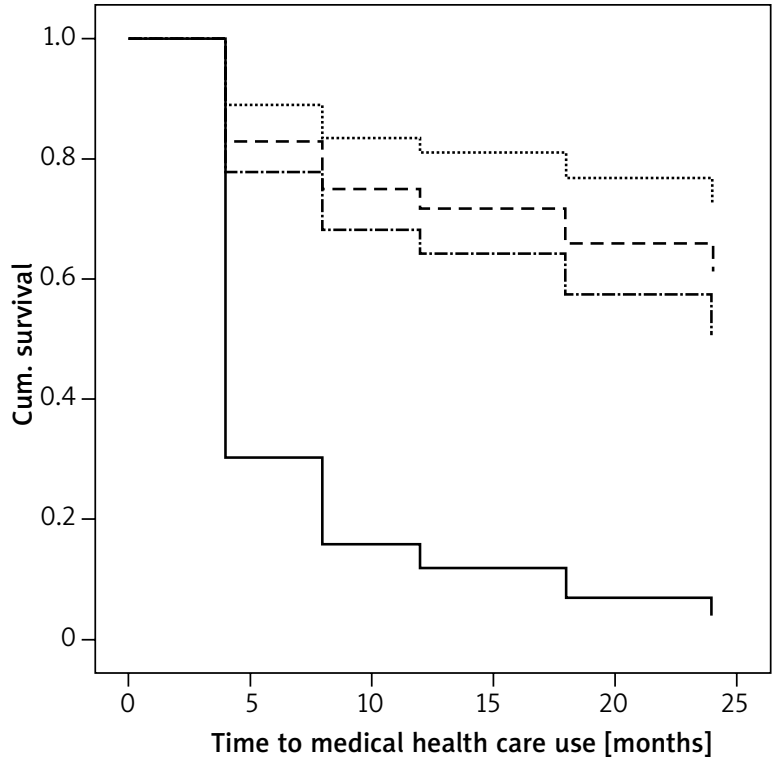

(Overall model score: $X 2(3)=19,031 . P<0.001,-2 \mathrm{LL}=373,9)$, reference value - poor responder, HR (moderate responder) $=0.26(p=0.04,95 \% \mathrm{Cl}: 0.072-$ 0.937 ), HR (good responder) $=0.168(p<0.001,95 \% \mathrm{Cl}: 0.067-0.462), \mathrm{HR}$ (excellent responder) $=0.117(p=0.01,95 \% \mathrm{Cl}: 0.023-0.599)$. GETE - global evaluation of treatment effectiveness, HR - hazard ratio.

\section{GETE (investigator response assessment):}

$\neg$ Poor responder (non-responder)

Moderate responder (non-responder)

- $\mathbf{r}$ ? Good responder (responder)

.....: Excellent responder (responder)

Figure 3. Effect of physician's global evaluation of treatment effectiveness (GETE) on medical healthcare use risk calculated by Cox regression $(n=112)$

use risk was analysed and significant risk reduction was seen for excellent responders (88.3\%), good (83.2\%) and moderate responders (74.0\%). These results indicate both a clinical benefit and an overall economic benefit (direct medical cost savings in healthcare utilisation and improvement at work productivity) [35]. Only 1 patient reported SAE that was considered to be study treatment related. The frequency of SAEs reported in the Czech Republic subgroup population (5.4\%) was similar to that reported in the global expeRience registry $(6.9 \%)$ and various clinical studies (3\% to $6 \%$ ) $[25,36,37]$. The single death reported was not related to omalizumab.

The limitation of this Czech Republic sub-population analysis is the low patient number for data analysis, in addition to limitations mentioned previously for the overall expeRience registry [13]. Moreover, demographic and clinical factors other than the treatment of interest may contribute to the observed effects in observational studies. Perceived physician bias in patient selection and continuation on omalizumab therapy beyond 16 weeks could have influenced the outcomes (for example, physicians were not blinded to the treatment). Another limiting factor could be the relatively infrequent data collection. 
Despite these potential obstacles, the present findings are in line with those from the randomised controlled trials, and statistical power was further invigorated by the repeated measures design. Thus, the improvement in clinical outcomes observed here is very likely due to omalizumab treatment. Since this study utilises an observational registry, there was no placebo arm; it is therefore possible that some of the improvements seen with omalizumab treatment may be partly related to a placebo effect and/or to the closer monitoring of the patients enrolled in the registry. Another limitation is that patients' baseline data were collected retrospectively from their medical records. Nevertheless, the baseline characteristics were similar to those in the global registry [13].

\section{Conclusions}

The results from the Czech Republic subgroup analysis are supportive of the existing evidence that omalizumab add-on therapy improves lung function and asthma control and reduces OCS use, exacerbations, healthcare utilisation and absence from work in patients with uncontrolled persistent allergic asthma [14]. These findings are likely to be relevant to the positive impact of omalizumab on the direct and indirect healthcare costs and in reducing the socioeconomic burden of uncontrolled allergic asthma in the Czech Republic. Patients' assessments and omalizumab administration are performed at the network of National Centres for Severe Asthma. Such specialized centres should be made accessible to the patients with uncontrolled allergic asthma for optimal care. GETE assessment is an effective predictor of long-term response to omalizumab treatment in the Czech Republic.

\section{Acknowledgments}

The registry was designed and sponsored by Novartis Pharma AG. The authors thank Vasavi Yada and Kunal Jain of Novartis for providing professional medical writing support, which was funded by Novartis Pharma AG in accordance with Good Publication Practice (GPP3) guidelines. The authors thank the patients who participated and the staff at the participating clinical centres. The members of the NCTA are: Dr. Bronislava Novotná (University Hospital Brno), Prof. Petr Panzner (University Hospital Plzeň) and Prof. Martina Vašáková (Thomayer Hospital Praha).

\section{Conflict of interest}

Ruzickova-Kirchnerova O. has been paid lecture fees by Mundipharma and Novartis. Valena T. is an employee of Novartis. Novosad J. has been paid lecture fees by Boehringer Ingelheim, Novartis and AstraZeneca. Terl M. has served on scientific advisory boards for AstraZeneca,
GSK, Novartis and TEVA, and has been paid lecture fees by AstraZeneca, Berlin-Chemie, Boehringer Ingelheim, GSK, Novartis, Stallergenes and TEVA.

\section{References}

1. Global strategy for asthma management and prevention. 2018. Available from: http://ginasthma.org/2018-gina-report-global-strategy-for-asthma-management-and-prevention/. Accessed 23 March 2018.

2. Jarvis D, Newson R, Lotvall J, et al. Asthma in adults and its association with chronic rhinosinusitis: the GA2LEN survey in Europe. Allergy 2012; 67: 91-8.

3. Tuberculosis and respiratory diseases 2014. Available from: http://uzis.cz/system/files/tbc2014.pdf. Accessed 23 March 2018.

4. Peters SP. Safety of inhaled corticosteroids in the treatment of persistent asthma. J Natl Med Assoc 2006; 98: 851-61.

5. Rabe KF, Adachi M, Lai CK, et al. Worldwide severity and control of asthma in children and adults: the global asthma insights and reality surveys. J Allergy Clin Immunol 2004; 114: 40-7.

6. Bousquet J, Siergiejko Z, Swiebocka E, et al. Persistency of response to omalizumab therapy in severe allergic (IgE-mediated) asthma. Allergy 2011; 66: 671-8.

7. Humbert M, Beasley R, Ayres J, et al. Benefits of omalizumab as add-on therapy in patients with severe persistent asthma who are inadequately controlled despite best available therapy (GINA 2002 step 4 treatment): INNOVATE. Allergy 2005; 60: 309-16.

8. Siergiejko Z, Swiebocka E, Smith N, et al. Oral corticosteroid sparing with omalizumab in severe allergic (IgE-mediated) asthma patients. Curr Med Res Opin 2011; 27: 2223-8.

9. Deschildre A, Marguet C, Salleron J, et al. Add-on omalizumab in children with severe allergic asthma: a 1-year real life survey. Eur Respir J 2013; 42: 1224-33.

10. Korn S, Thielen A, Seyfried S, et al. Omalizumab in patients with severe persistent allergic asthma in a real-life setting in Germany. Respir Med 2009; 103: 1725-31.

11. Barnes N, Menzies-Gow A, Mansur AH, et al. Effectiveness of omalizumab in severe allergic asthma: a retrospective UK real-world study. J Asthma 2013; 50: 529-36.

12. Kuprys-Lipinska I, Molinska K, Kuna P. The effect of omalizumab on eosinophilic inflammation of the respiratory tract in patients with allergic asthma. Pneumonol Alergol Pol 2016; 84: 232-43.

13. Braunstahl GJ, Chen CW, Maykut R, et al. The eXpeRience registry: the 'real-world' effectiveness of omalizumab in allergic asthma. Respir Med 2013; 107: 1141-51.

14. Braunstahl GJ, Leo J, Thirlwell J, et al. Uncontrolled persistent allergic asthma in practice: eXpeRience registry baseline characteristics. Curr Med Res Opin 2011; 27: 761-7.

15. Summary of Product Characteristics [http://www.ema.europa.eu/docs/en_GB/document_library/EPAR_-_Product_Information/human/000606/WC500057298.pdf]. Accessed 23 March 2018.

16. Ehrs PO, Nokela M, Stallberg B, et al. Brief questionnaires for patient-reported outcomes in asthma: validation and usefulness in a primary care setting. Chest 2006; 129: 925-32.

17. Nathan RA, Sorkness CA, Kosinski M, et al. Development of the asthma control test: a survey for assessing asthma control. J Allergy Clin Immunol 2004; 113: 59-65.

18. Lloyd A, Turk F, Leighton T, Canonica GW. Psychometric evaluation of global evaluation of treatment effectiveness: 
a tool to assess patients with moderate-to-severe allergic asthma. J Med Econ 2007; 10: 285-96.

19. Schatz M, Kosinski M, Yarlas AS, et al. The minimally important difference of the Asthma Control Test. J Allergy Clin Immunol 2009; 124: 719-23 e1.

20. Juniper EF, Guyatt GH, Cox FM, et al. Development and validation of the Mini Asthma Quality of Life Questionnaire. Eur Respir J 1999; 14: 32-8.

21. Juniper EF, Guyatt GH, Willan A, Griffith LE. Determining a minimal important change in a disease-specific Quality of Life Questionnaire. J Clin Epidemiol 1994; 47: 81-7.

22. Sedlák V, Chlumský J, Teřl M, et al. Doporučený postup diagnostiky a léčby obtižně léčitelného bronchiálního astmatu. 2016. Available from: http://www.pneumologie.cz/guidelines. Accessed 23 March 2018 (National Severe Asthma Guidelines in Czech).

23. Terl M, Sedlak V, Cap P, et al. Asthma management: a new phenotype-based approach using presence of eosinophilia and allergy. Allergy 2017; 72: 1279-87.

24. Kerstjens HA, Engel M, Dahl R, et al. Tiotropium in asthma poorly controlled with standard combination therapy. N Engl J Med 2012; 367: 1198-207.

25. Schumann C, Kropf C, Wibmer T, et al. Omalizumab in patients with severe asthma: the XCLUSIVE study. Clin Respir J 2012; 6: 215-27.

26. Brusselle G, Michils A, Louis R, et al. "Real-life" effectiveness of omalizumab in patients with severe persistent allergic asthma: the PERSIST study. Respir Med 2009; 103: 1633-42.

27. Cazzola M, Camiciottoli G, Bonavia M, et al. Italian real-life experience of omalizumab. Respir Med 2010; 104: 1410-6.

28. Menzella F, Galeone C, Formisano D, et al. Real-life efficacy of omalizumab after 9 years of follow-up. Allergy Asthma Immunol Res 2017; 9: 368-72.

29. Kuprys-Lipinska I, Majak P, Molinska J, Kuna P. Effectiveness of the Polish program for the treatment of severe allergic asthma with omalizumab: a single-center experience. BMC Pulm Med 2016; 16: 61.

30. Ledford D, Busse W, Trzaskoma B, et al. A randomized multicenter study evaluating Xolair persistence of response after long-term therapy. J Allergy Clin Immunol 2017; 140: 162-9.e2.

31. Kuprys-Lipinska I, Kuna P. Loss of asthma control after cessation of omalizumab treatment: real life data. Postep Dermatol Alergol 2014; 31: 1-5.

32. Hutyrova B, Bystron J, Chlumsky J, et al. Effect of omalizumab treatment on allergic comorbidities in patients with severe allergic asthma - real life experience from Czech AntiIgE Registry. Eur Respir J 2017; 50 (Suppl 61): PA3541.

33. Novelli F, Latorre M, Vergura L, et al. Asthma control in severe asthmatics under treatment with omalizumab: a crosssectional observational study in Italy. Pulm Pharmacol Ther 2015; 31: 123-9.

34. Bardelas J, Figliomeni M, Kianifard F, Meng X. A 26-week, randomized, double-blind, placebo-controlled, multicenter study to evaluate the effect of omalizumab on asthma control in patients with persistent allergic asthma. J Asthma 2012; 49: 144-52.

35. Braunstahl GJ, Canvin J, Peachey G, et al. Healthcare resource utilization in patients receiving omalizumab for allergic asthma in a real-world setting. Biol Ther 2014; 4: 57-67.

36. Corren J, Casale TB, Lanier B, et al. Safety and tolerability of omalizumab. Clin Exp Allergy 2009; 39: 788-97.

37. Milgrom H, Fowler-Taylor A, Vidaurre CF, Jayawardene S. Safety and tolerability of omalizumab in children with allergic (IgE-mediated) asthma. Curr Med Res Opin 2011; 27: 163-9. 